\title{
WEIGHTED BRIANCHON-GRAM DECOMPOSITION
}

\author{
JOSÉ AGAPITO
}

\begin{abstract}
We give in this note a weighted version of Brianchon-Gram's decomposition for a simple polytope. We can derive from this decomposition the weighted polar formula of $[\mathrm{A}$ and a weighted version of Brion's theorem [Br1], in a manner similar to $[\mathrm{H}$, where the unweighted case is worked out. This weighted version of Brianchon-Gram is a direct consequence of the ordinary Brianchon-Gram formula.
\end{abstract}

\section{INTRODUCTION}

Let $A \subset \mathbb{R}^{n}$ be a closed convex subset. The characteristic function $\mathbf{1}_{A}$ of $A$ is the function $\mathbf{1}_{A}: \mathbb{R}^{n} \rightarrow \mathbb{C}$ given by

$$
\mathbf{1}_{A}(x)=\left\{\begin{array}{ll}
1 & \text { if } x \in A \\
0 & \text { if } x \notin A
\end{array} .\right.
$$

Let $\mathcal{K}\left(\mathbb{R}^{n}\right)$ be the complex vector space spanned by the functions $\mathbf{1}_{A}$. Thus, a function $f \in \mathcal{K}\left(\mathbb{R}^{n}\right)$ is a linear combination

$$
f=\sum_{i=1}^{m} \alpha_{i} \mathbf{1}_{A_{i}},
$$

where the $A_{i}$ are closed convex sets in $\mathbb{R}^{n}$ and the $\alpha_{i}$ are complex numbers.

Among the elements of $\mathcal{K}\left(\mathbb{R}^{n}\right)$ there are three well known decomposition formulas: the Brianchon-Gram decomposition $[\mathrm{Br}, \mathrm{G}]$ (see also $[\mathrm{B}]$ and $[\mathrm{S}]$ ), which determines the characteristic function of any polytope as a signed sum of characteristic functions of cones associated to its faces, the polar decomposition of a simple polytope $P([\mathrm{~L},[\mathrm{~V}])$, which uses the notion of polarization and Brianchon-Gram's formula in order to write the characteristic function of $P$ in terms of the characteristic functions of cones based on the vertices of $P$ only, and the Brion decomposition of a polytope [Br1], which is also a direct consequence of Brianchon-Gram's formula.

2000 Mathematics Subject Classification. Primary 52B.

Partially supported by NSF (United States) through grants DMS 99/71914 and DMS 04/05670 and by FCT (Portugal) through program POCTI/FEDER and grant POCTI/SFRH/BPD/20002/2004. 
We can put weights to the faces of $P$ in a meaningful way and get a new element of $\mathcal{K}\left(\mathbb{R}^{n}\right)$. For instance, let $q$ be any complex number and let $[a, b]$ be any interval. We can write (see Figure 1)

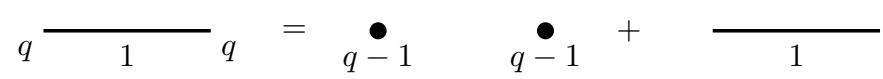

FiguRE 1. Decomposition of an interval.

That is, as an element of $\mathcal{K}(\mathbb{R})$, the function $(q-1) \mathbf{1}_{\{a\}}+(q-1) \mathbf{1}_{\{b\}}+\mathbf{1}_{[a, b]}$ defines a weighted characteristic function over $[a, b]$. We denote it by $\mathbf{1}_{[a, b]}^{q}$. In general, we can assign arbitrary complex values to the facets of a polytope $P$ and construct a new element $\mathbf{1}_{P}^{w}$ of $\mathcal{K}\left(\mathbb{R}^{n}\right)$. (See (3).)

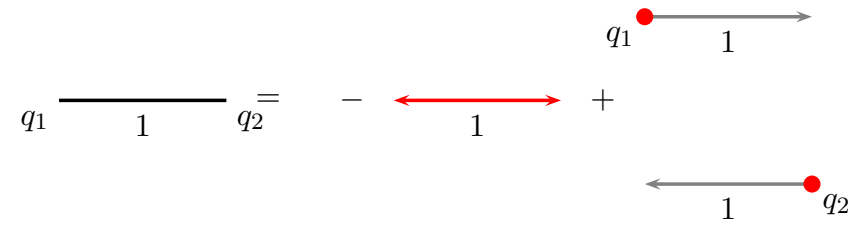

FiguRE 2. Weighted Brianchon-Gram decomposition for an interval.

When we assign the same value $q$ to the facets of $P$, we also denote $\mathbf{1}_{P}^{w}$ by $\mathbf{1}_{P}^{q}$. The weighted polar decomposition formula of [A] expresses $\mathbf{1}_{P}^{q}$ as an alternating sum of weighted characteristic functions of cones based on the vertices of $P$. For example, in the case of $\mathbf{1}_{[a, b]}^{q}$, we have $\mathbf{1}_{[a, b]}^{q}=\mathbf{1}_{[a, \infty)}^{q}-$ $\mathbf{1}_{(-\infty, b]}^{1-q}$. The purpose of this note is to show that $\mathbf{1}_{P}^{w}$ (defined in (3) ) satisfies a weighted version of Brianchon-Gram's formula, from which it readily follows the weighted polar formula of [A] and a weighted version of Brion's theorem [Br1]. The relationship among these formulas has already been pointed out by Haase [H] in the unweighted case. Our main result is the weighted version of Brianchon-Gram's formula as stated in (4). (See Figures 2, 3] and 4] for illustrations of this formula.)

\section{The Weighted FORMulA}

Let $P$ be a $d$-dimensional polyhedron in $\mathbb{R}^{d}$ (for standard definitions on polyhedra we refer to $[\mathrm{B}]$ ). We can write it as the intersection of a finite number of half-spaces

$$
P=H_{1} \cap \ldots \cap H_{N}
$$


where $H_{i}=\left\{x \mid\left\langle u_{i}, x\right\rangle+\mu_{i} \geq 0\right\}$, with $\mu_{i} \in \mathbb{R}$ and $u_{i} \in\left(\mathbb{R}^{d}\right)^{*}$ for $1 \leq i \leq N$. Note that $\mathbb{R}^{d}=\{x \mid\langle 0, x\rangle+0 \geq 0\}$, hence $\mathbb{R}^{d}$ is trivially a polyhedron. It follows that $P$ is a closed convex set. We assume that $P$ is obtained with the smallest possible $N$. The facets of $P$ are $\sigma_{i}=P \cap \partial H_{i}$ for $i=1, \ldots, N$. If the intersection (11) is bounded then $P$ is a polytope. We say that a $d$-dimensional polyhedron $P$ is simple if every vertex of $P$ belongs (when it exists) to exactly $d$ facets of $P$. In the case of a polyhedron without vertices, we assume that this condition is trivially satisfied.

Let $P$ be any $d$-dimensional polyhedron in $\mathbb{R}^{d}$. For each $i=1, \ldots, N$, we assign arbitrary complex numbers $q_{i}$ to the facets $\sigma_{i}$ of $P$. Each non-trivial face $F$ of $P(F \neq \phi, P)$ can be uniquely described as an intersection of facets

$$
F=\bigcap_{i \in I_{F}} \sigma_{i}
$$

where $I_{F}$ denotes the set of all facets of $P$ containing $F$. When $P$ is simple, the number of elements in $I_{F}$ is equal to the codimension of $F$.

To each non-trivial face $F$ we assign the value $\prod_{i \in I_{F}} q_{i}$. When $F=P$, we give it the value 1 . This amounts to defining the weighted function $w: P \rightarrow \mathbb{C}$ by $w(x)=\prod_{i \in I_{F}} q_{i}$, where $F$ is the face of $P$ of smallest dimension containing $x$. If $x$ is in the interior of $P$, we set $w(x)=1$. We extend this definition to all $\mathbb{R}^{d}$ and get the weighted characteristic function

$$
\mathbf{1}_{P}^{w}(x)=\left\{\begin{array}{cl}
w(x) & \text { if } x \in P \\
0 & \text { if } x \notin P
\end{array} .\right.
$$

Now, let $F$ be any face of $P$. The tangent cone to $P$ at $F$ is

$$
\mathbf{C}_{F}=\{y+r(x-y) \mid r \geq 0, y \in F, x \in P\} .
$$

It follows that $\mathbf{C}_{F}$ is also a polyhedron. For example, when $F=P$, we have $\mathbf{C}_{F}=\mathbb{R}^{d}$.

Theorem 1 (Weighted Brianchon-Gram). Let $P$ be a simple polytope of $d i$ mension $d$ in $\mathbb{R}^{d}$. We have

$$
\mathbf{1}_{P}^{w}=\sum_{F \preceq P}(-1)^{\operatorname{dim} F} \mathbf{1}_{\mathbf{C}_{F}}^{w},
$$

where the sum is over all faces $F$ of $P$.

When $q_{1}=\ldots=q_{N}=1$, we have the ordinary Brianchon-Gram formula. We illustrate this theorem for a triangle in Figure 3. (See also Figure 2]) 


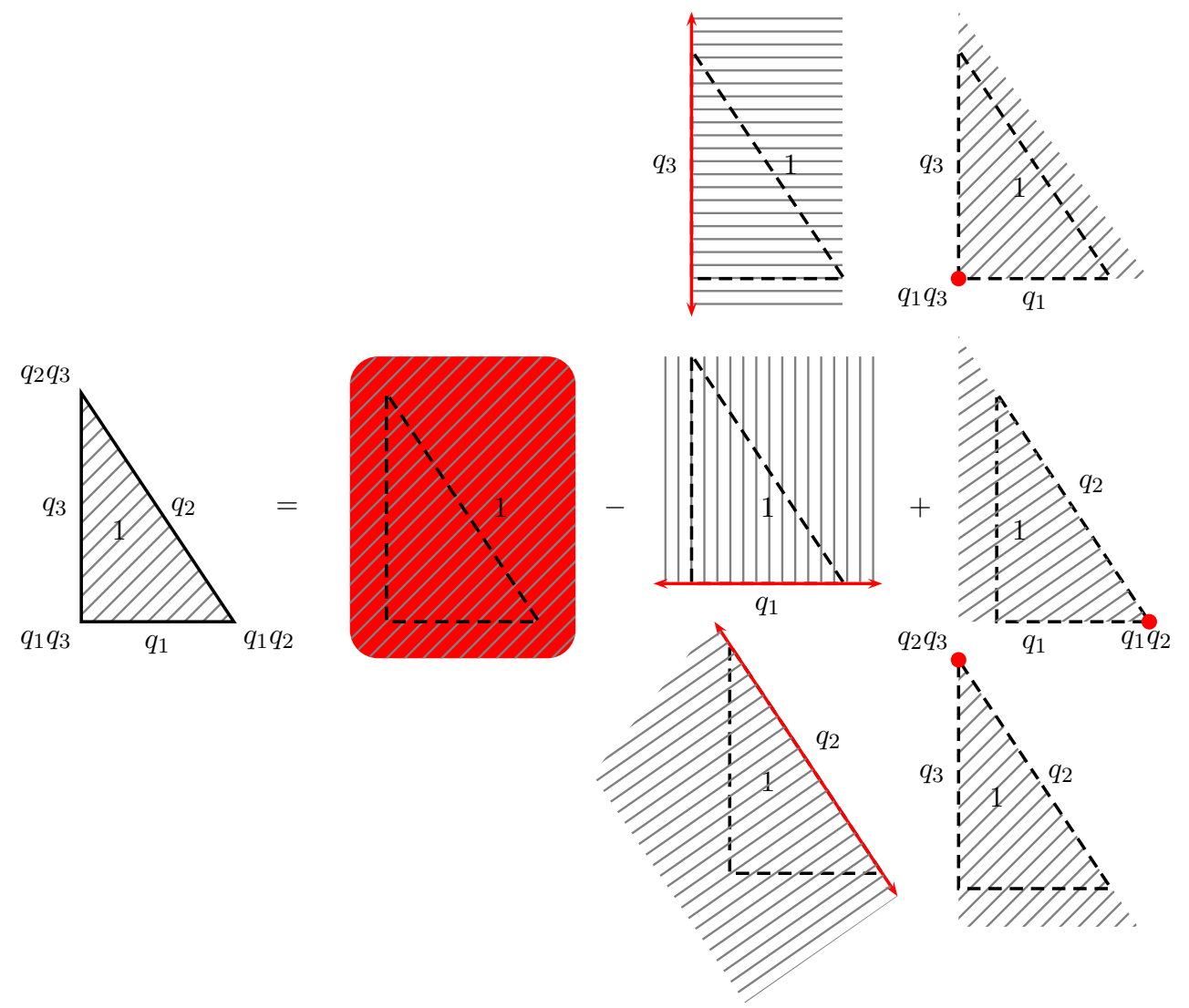

Figure 3. Weighted Brianchon-Gram decomposition for a triangle.

Proof. Let $\Sigma$ be the set of facets of $P$ and let $F$ be a proper face of $P$. Let $I_{F}=\{\sigma \in \Sigma \mid F \subset \sigma\}$. Then $F=\bigcap_{i \in I_{F}} \sigma_{i}$. Since $P$ is simple, the cardinality of $I_{F}$ is equal to the codimension of $F$ and for all possible non-empty subsets $J$ of $I_{F}$, the face $\bigcap_{i \in J} \sigma_{i}$ of $P$ contains $F$. A straightforward computation shows that

$$
\prod_{i \in I_{F}} q_{i}=1+\sum_{\phi \neq J \subset I_{F}} \prod_{i \in J}\left(q_{i}-1\right)
$$

where the $q_{i}$ are arbitrary complex numbers assigned to the facets $\sigma_{i}$ of $P$. We decompose $P$ into all its faces $F$ (including $F=P$ ). By (3) and (5), we have

$$
\mathbf{1}_{P}^{w}=\mathbf{1}_{P}+\sum_{F \neq P} \prod_{i \in I_{F}}\left(q_{i}-1\right) \mathbf{1}_{F}
$$


We can apply the ordinary Brianchon-Gram formula to each $F$ and get

$$
\mathbf{1}_{P}^{w}=\sum_{F \preceq P}(-1)^{\operatorname{dim} F} \mathbf{1}_{\mathbf{C}_{F}}+\sum_{F \neq P} \prod_{i \in I_{F}}\left(q_{i}-1\right) \sum_{G \preceq F}(-1)^{\operatorname{dim} G_{1}} \mathbf{1}_{\mathbf{C}_{G}} .
$$

Note that (6) also holds for the tangent cones $C_{F}$ of $P$ at $F$; that is

$$
\mathbf{1}_{\mathbf{C}_{F}}^{w}=\mathbf{1}_{\mathbf{C}_{F}}+\sum_{H \neq \mathbf{C}_{F}} \prod_{i \in I_{H}}\left(q_{i}-1\right) \mathbf{1}_{H}
$$

Since the faces $H$ of the tangent cone $\mathbf{C}_{F}$ are in turn tangent cones associated to the faces $G$ of $F$, we can regroup the characteristic functions in (7) according to (8) and obtain

$$
\mathbf{1}_{P}^{w}=\sum_{F \preceq P}(-1)^{\operatorname{dim} F} \mathbf{1}_{\mathbf{C}_{F}}^{w}
$$

Theorem 1 can be extended to non-simple polytopes where the only faces $F$ which are non-generic (i.e. $F$ has dimension $f$ but $\left|I_{F}\right| \neq d-f$ ) are vertices.

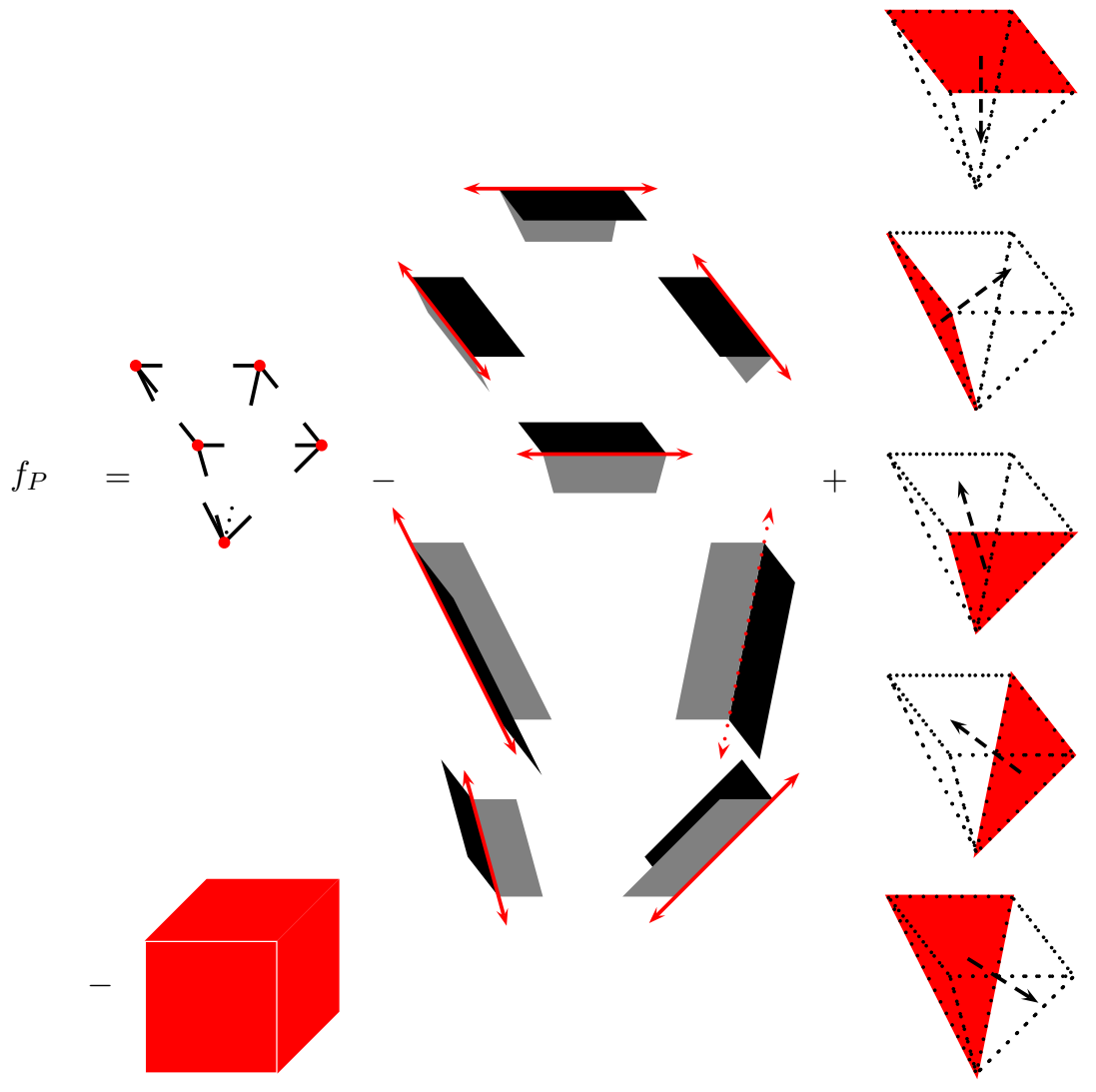

FIGURE 4. Weighted Brianchon-Gram decomposition for a pyramid. 
Indeed, in dimension three, only vertices may be non-generic for an arbitrary non-simple polytope. In higher dimensions though, there may be other faces which are the intersection of too many facets. We illustrate this special extension for a pyramid in Figures 456 and 7

Let $P$ be any non-simple polytope of dimension $d$ in $\mathbb{R}^{d}$ whose non-generic faces are only vertices. Let $V_{n s}(P)$ be the set of non-simple vertices of $P$. If $v \in V_{n s}(P)$, then $\left|I_{v}\right|>d$. (Recall that in the simple case $\left|I_{v}\right|=d$ for all vertices of $P$.)

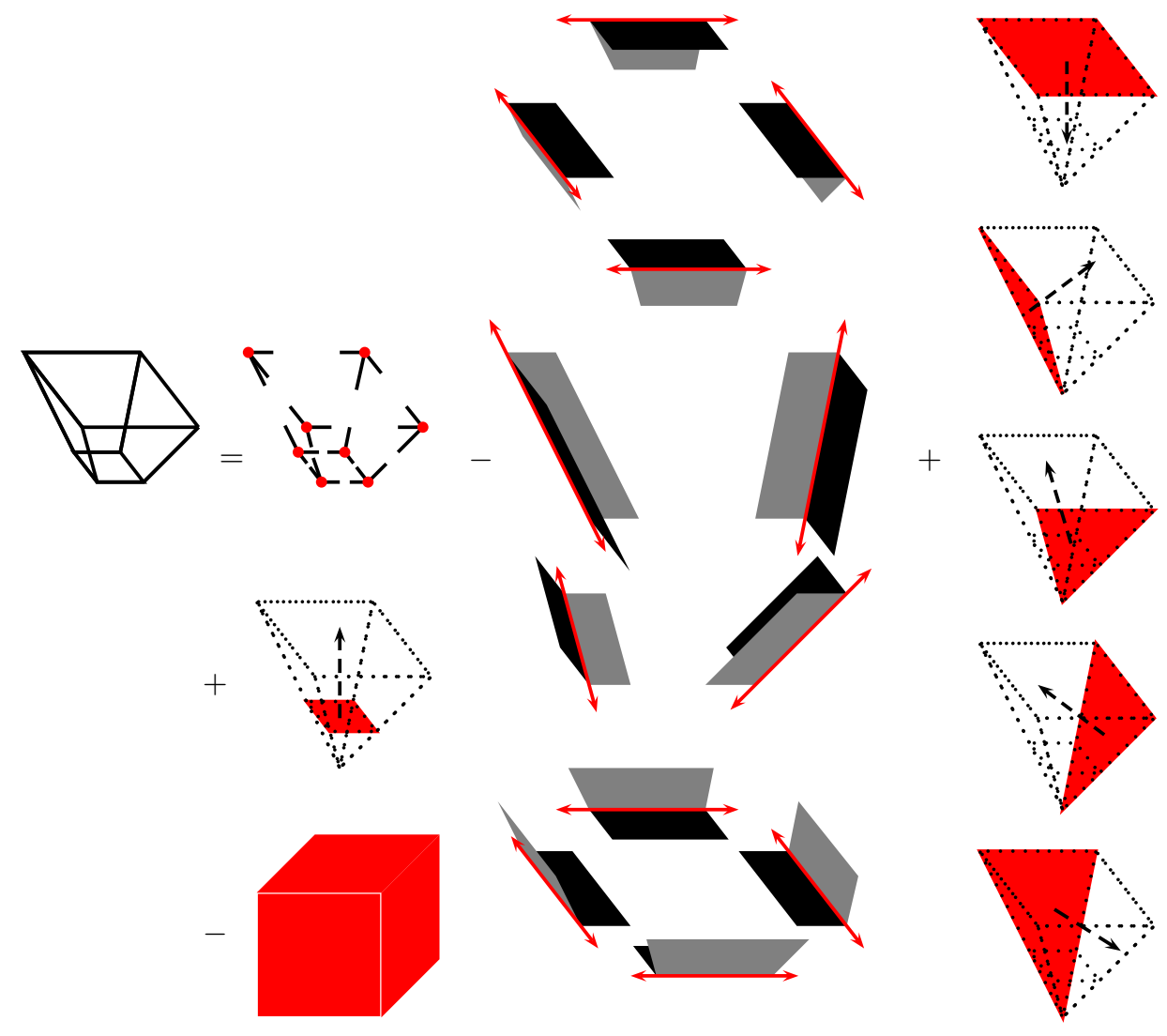

FiguRE 5. Weighted Brianchon-Gram for a truncated pyramid.

We chop off all the non-simple vertices $v$ of $P$ by taking hyperplanes $\sigma_{v}$ very close to these vertices. We orient the $\sigma_{v}$ away from $v$ and denote the corresponding half-space associated to $\sigma_{v}$ by $H_{v}$. We assign the constant value 1 to the hyperplanes $\sigma_{v}$. We obtain a simple polytope $P_{s}$ for which formula (4) 
holds; that is

$$
\mathbf{1}_{P_{s}}^{w}=\sum_{F_{s} \preceq P_{s}}(-1)^{\operatorname{dim} F_{s}} \mathbf{1}_{\mathbf{C}_{F_{s}}}^{w},
$$

where the sum is over all the faces $F_{s}$ of $P_{s}$. On the other hand, we set

$$
f_{P}=\sum_{F \preceq P}(-1)^{\operatorname{dim} F} \mathbf{1}_{\mathbf{C}_{F}}^{w},
$$

where the sum is now over all the faces $F$ of the non-simple polytope $P$.

We can clearly see that $\mathbf{1}_{\mathbf{C}_{F}}^{w}=\mathbf{1}_{\mathbf{C}_{F_{s}}}^{w}$ for all the faces of $P$ and $P_{s}$ which are not the non-simple vertices $v \in V_{n s(P)}$ and are not the faces of $P_{s}$ contained in the various $\sigma_{v}$. Thus, we have

$$
\mathbf{1}_{P_{s}}^{w}-f_{P}=\sum_{v \in V_{n s}(P)}\left(\sum_{F_{s} \subset \sigma_{v}}(-1)^{\operatorname{dim} F_{s}} \mathbf{1}_{\mathbf{C}_{F_{s}}}^{w}-\mathbf{1}_{\mathbf{C}_{v}}^{w}\right) .
$$

Notice that all the cross sections of $\mathbf{C}_{v}$ contained in $H_{v}$ and parallel to $\sigma_{v}$, are simple polytopes (of the same type) in $\mathbb{R}^{d-1}$. This is a consequence of the imposed condition on $P$, that its only non-generic faces be vertices. We can apply the weighted Brianchon-Gram formula to these cross sections and get weighted characteristic functions over its faces, which are equal in absolute value but with opposite signs, to the weighted characteristic functions over the faces of the corresponding cross sections of the cones $\mathbf{C}_{F_{s}}$, where $F_{s} \subset \sigma_{v}$, for all $v \in V_{n s}(P)$. Then, we can write (11) as

$$
\mathbf{1}_{P_{s}}^{w}-f_{P}=-\sum_{v \in V_{n s}(P)} \mathbf{1}_{\mathbf{C}_{v} \backslash H_{v}}^{w} .
$$
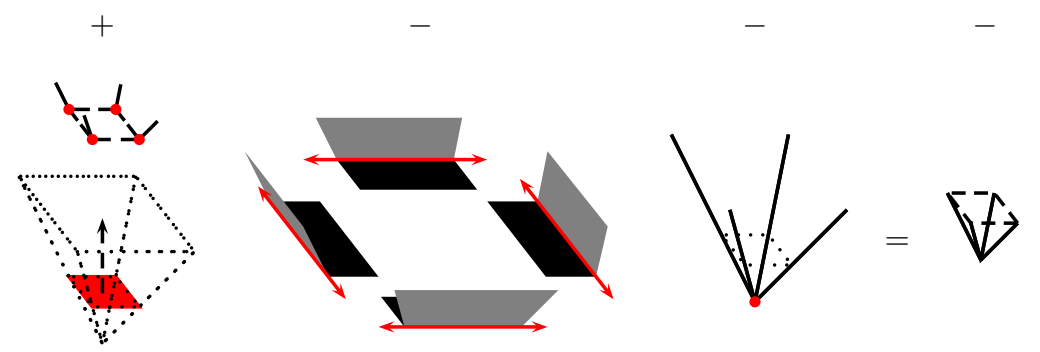

Figure 6 . Key difference $\mathbf{1}_{P_{s}}^{w}-f_{P}$ for a pyramid.

On the other hand, it can be easily checked that

$$
\mathbf{1}_{P_{s}}^{w}-\mathbf{1}_{P}^{w}=-\sum_{v \in V_{n s}(P)} \mathbf{1}_{\mathbf{C}_{v} \backslash H_{v}}^{w} .
$$




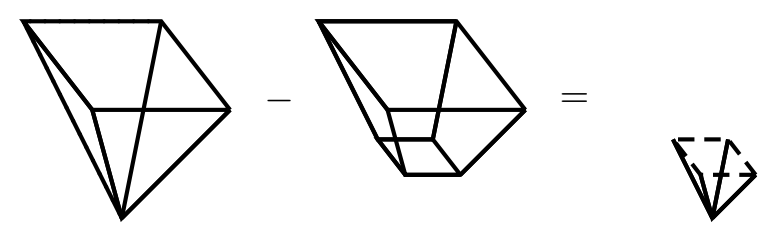

FiguRE 7. Difference of the pyramid and the truncated pyramid.

Therefore, we conclude that

$$
\mathbf{1}_{P}^{w}=\sum_{F \preceq P}(-1)^{\operatorname{dim} F} \mathbf{1}_{\mathbf{C}_{F}}^{w} .
$$

As an immediate consequence of Theorem 1 we obtain a weighted version of Brion's theorem ${ }^{1}$ Br1. (See also Br2.)

Corollary 1 . For any simple polytope $P$, we have

$$
\mathbf{1}_{P}^{w}=g+\sum_{v} \mathbf{1}_{\mathbf{C}_{v}}^{w}
$$

where $g$ is a linear combination of characteristic functions of cones with straight lines and the sum is over all vertices $v$ of $P$.

This readily follows from grouping together all tangent cones in (4) that contain straight lines, just as in $[\mathrm{H}]$.

When $P$ is a simple polyhedron and the same value $q \in \mathbb{C}$ is assigned to all its facets, the weighted characteristic function (3) gets the form

$$
\mathbf{1}_{P}^{w}(x)=\mathbf{1}_{P}^{q}(x)=\left\{\begin{array}{cl}
q^{\operatorname{codim}(F)} & \text { if } x \in P \\
0 & \text { if } x \notin P
\end{array},\right.
$$

where $F$ is the face of $P$ of smallest dimension containing $x$. Theorem 1 implies the weighted polytope decomposition of $[\mathrm{A}$. To show this, let $\xi$ be a polarizing vector ${ }^{2}$ in $\left(\mathbb{R}^{d}\right)^{*}$ and let $\Delta$ be a simple polytope in $\mathbb{R}^{d}$. (We now follow the definitions and notation of $[\mathrm{A}$.) We put together the faces of $P$ according to where they achieve their minimum in the $\xi$-direction. We obtain

$$
(-1)^{\# v} \mathbf{1}_{\mathbf{C}_{v}^{\sharp}}^{w_{v}}=\sum_{\substack{v \preceq F \preceq P \\ \xi(v) \leq \xi(F)}}(-1)^{\operatorname{dim} F} \mathbf{1}_{\mathbf{C}_{F}}^{q},
$$

\footnotetext{
${ }^{1}$ Brion already proved a more general weighted version of his formula in [Br1] (see page 82 of Br2]

2 a polarizing vector is a generic element of $\left(\mathbb{R}^{n}\right)^{*}$ which is nonconstant on each edge of $P$. (See $[\mathrm{L}, \mathrm{V}]$ and compare with $[\mathrm{A}]$.)
} 
where $\# v$ denotes the number of edges of $\mathbf{C}_{v}$ flipped according to $\xi$ and where $\mathbf{1}_{\mathbf{C}_{v}^{\sharp}}^{w_{v}}$ is the weighted characteristic function of the $\xi$-polarized tangent cone $\mathbf{C}_{v}^{\sharp}$ defined in $[\mathrm{A}$. (To agree with the weighted formulas in $\mathrm{A}$ ] we use the substitution $q=1 /(1+y)$.) Then

$$
\sum_{v}(-1)^{\# v} \mathbf{1}_{\mathbf{C}_{v}^{\sharp}}^{w_{v}}=\sum_{v} \sum_{\substack{v \preceq F \preceq P \\ \xi(v) \leq \xi(F)}}(-1)^{\operatorname{dim} F} \mathbf{1}_{\mathbf{C}_{F}}^{q}=\sum_{F}(-1)^{\operatorname{dim} F} \mathbf{1}_{\mathbf{C}_{F}}^{q}=\mathbf{1}_{P}^{q} .
$$

Thus, the weighted polar decomposition of [A] is a direct consequence of Theorem 1] which can be proved exactly in the same fashion as in [L].

\section{REFERENCES}

[A] J. Agapito, A weighted version of quantization commutes with reduction for a toric manifold. To appear in the Proceedings of the Summer Research Conference on Integer Points in Polyhedra, Snowbird (2003). To be published in Contemporary Mathematics.

[B] A. Barvinok, A Course in Convexity, Graduate Studies in Mathematics 54, American Mathematical Society (2002).

[Br] C. J. Brianchon, Théorème nouveau sur les polyèdres, J. Ecole Polytechnique, 15 (1837), 317-319.

[Br1] M. Brion, Points entiers dans les polyèdres convexes, Ann. Sci. École Norm. Sup. (4) 21 (1988), 653-663.

[Br2] M. Brion, Polyèdres et réseaux, Enseign. Math (2) 38 (1992), 71-88.

[G] J.P. Gram, Om rumvinklerne $i$ et polyeder, Tidsskrift for Math. (Copenhagen), (3) 4 (1874), 161-163.

[H] C. Haase, Polar decomposition and Brion's theorem, Preprint (December 2003), ArXiv: math.CO/0312095

[L] J. Lawrence, Polytope volume computation, Math. Comp. (195) 57 (1991), 259-271.

[S] G.C. Shephard, An elementary proof of Gram's theorem for convex polytopes, Canad. J. Math. 19 (1967), 1214-1217.

[V] A.N. Varchenko, Combinatorics and topology of the arrangement of affine hyperplanes in the real space, (English translation) Funct. Anal. Appl. (1) 21 (1987), 9-19.

DEPARTAMENTO DE MATEMÁTICA, INSTITUTO SUPERIOR TÉCNICO, AV. ROVISCO PAIS, 1049-001 LISBON, PORTUGAL, FAX: (351) 218417035

E-mail address: agapito@math.ist.utl.pt 